\title{
A formative study exploring parents' and health visitor's perspectives of the relevance and acceptability of weight management programmes targeting preschool children in Grampian, Scotland
}

\author{
F. Douglas ${ }^{1}$, J. Clark ${ }^{2,3}$, L. C. A. Craig ${ }^{2}$, A. E. Gilmartin ${ }^{2}$, C. Commerford ${ }^{3}$ and G. McNeill ${ }^{1,2}$ \\ ${ }^{1}$ Rowett Institute of Nutrition and Health, ${ }^{2}$ Institute of Applied Health Sciences, University of Aberdeen and \\ ${ }^{3}$ NHS Grampian, Aberdeen, UK
}

The UK Child Health Surveillance Programme (CHSP) reported that $8.6 \%$ of children born in Scotland in 2001 were obese $\left(\mathrm{BMI} \geqslant 95\right.$ th centile of the UK 1990 reference data) at 3.5 years of age compared to $7.9 \%$ of those born in $1995^{(1)}$. There is growing policy maker and health professional interest in preschool weight management interventions to halt or reverse this trend ${ }^{(2,3)}$. In 2012 , NHS Grampian commissioned formative research to inform the development of a pre-school weight management intervention planned for the region. Qualitative research was conducted with parents of pre-school aged children and health visitors (as the group of health professionals considered best placed to deliver such a programme) to explore their respective views and perceptions about the problem of obesity and overweight in this age group, and, the acceptability and feasibility of possible interventions. This paper presents the results of research which explored these issues with 34 parents and 15 health visitors based in the north-east of Scotland. The study took place between May 2012 and February 2013. Parents of preschool aged children were recruited by invitation through preschool nurseries, and health visitors were invited to participate through the community nursing service. Ethical approval was sought from and granted by the North of Scotland Research Ethics Committee. Nine focus groups were held with parents preschool children. The health visitors took part in individual in depth telephone interviews. Both the focus groups and interviews lasted between $45-90$ mins, were audio-recorded, transcribed verbatim and analysed thematically.

The research has revealed a rich picture of the variety of sources of information parents and healthy visitors use to make judgements about a pre-school age child's weight status, and the large areas of overlap and divergence that exist in those different perspectives. It also reveals interesting insights regarding parents' and health visitors' perspectives about the legitimacy and relevance of growth centile reference data and BMI charts in the context of 'real world' circumstances for this age group, and, the general resistance that exists amongst parents to the idea of programmes or health professional interventions targeting them about this issue. Despite parents' questioning of the relevance of a weight management programme for their preschool children, parents in this study described dealing with many significant challenges and pressures both within and from outside the family environment, as they struggle to 'get the balance right' in raising a healthy weight child. Furthermore, all parents who took part in this study expressed concern about their child's weight in the future. In particular parents described struggling with aspects of weaning and infant feeding.(e.g. deciding on portion size and over feeding), dealing with different perspectives within their families about appropriate foods and portion sizes for their children, managing different dietary preferences and energy requirements at family mealtimes and 'sticking to the healthy eating rules' when tired or feeling time pressured. This research also suggests that the development of (separate) visually-based resources for parents and health visitors, both to help assess a child's weight status may assist with problem recognition, and to depict age appropriate portion size for toddlers and infant, could be useful adjuncts to programmes intended for parents of children in this age group, and for health visitors attempting to work with parents in this area.

1. Scottish Public Health Observatory. 2007. Obesity in Scotland An epidemiology briefing. NHS National Services Scotland: Edinburgh.

2. The Scottish Government, 2010. Preventing Overweight and Obesity in Scotland: A Route Map Towards a Healthy Weight. Edinburgh: The Scottish Government.

3. Kerr A and Reid W, 2009. Update on work to achieve HEAT target 3: child healthy weight. HS paper 28/09. NHS Health Scotland. Available from: http://www.healthscotland.com/uploads/documents/9947-BDP2809.pdf. 